\title{
LIFTING THE ACTION OF A GROUP IN A FIBRE BUNDLE
}

BY T. E. STEWART ${ }^{1}$

Communicated by Deane Montgomery, January 8, 1960

1. Suppose that $B$ is a $G$-space for a given topological group $G$. That is we are given a continuous map $\bar{\alpha}: G \times B \rightarrow B$ satisfying the equations

$$
\begin{array}{ll}
\bar{\alpha}\left(u_{1} \cdot u_{2}, b\right)=\bar{\alpha}\left(u_{1}, \bar{\alpha}\left(u_{2}, b\right)\right), & u_{1}, u_{2} \in G, b \in B, \\
\bar{\alpha}(e, b)=b, & e \text { the identity of } G .
\end{array}
$$

Let $\Theta$ be a principal bundle over $B,[5$, p. 35] with total space $E$ and $H$ the structural group so that $B$ may be regarded as the orbit space of $E$ by $H$. We wish to consider here the problem of putting the two actions together in $E$ in a sense to be made precise below.

2. Let $B$ be a bundle with base $B$ and total space $E$. Suppose $B$ is a $G$-space given by a function $\bar{\alpha}$ as above. We say that action $(G, \bar{\alpha})$ can be lifted to $E$ in $B$ if $E$ can be given the structure of a $G$-space so that the projection of $E$ onto $B$ in $B$ is an equivariant map, i.e. so that if $\alpha$ gives the action of $G$ on $E$ we have the following commutative diagram:

$$
\begin{aligned}
& G \times E \stackrel{\alpha}{\rightarrow} E \\
& (1, p) \downarrow \quad \downarrow p \\
& G \times B \underset{\alpha}{\rightarrow} B
\end{aligned}
$$

$(G, \alpha)$ will then be called a lifting of the action $(G, \bar{\alpha})$. A lifting will be called a bundle lifting in $B$ if for each $u \in G$ the map $x \rightarrow \alpha(u, x)$ of $E$ onto $E$ is a bundle mapping.

For example, a group of diffeomorphisms of a manifold $B$ in the $C^{r}$-topology has a bundle lifting in the tangent bundle to $B$ in taking the differential of each element.

Proposition 2.1. If the action $(G, \bar{\alpha})$ on $B$ has a bundle lifting in the principal bundle $B$ with structural group $H$ and total space $E$, then $G \times H$ acts on $E$ in a canonical way. If $(G, \bar{\alpha})$ is a transitive action so is the action of $G \times H$. If the action $(G, \bar{\alpha})$ is free so is that of $G \times H$.

If $(G, \alpha)$ is the bundle lifting in $B$ of $(G, \bar{\alpha})$ define the action $(G \times H$, $\beta)$ in $E$ by $\beta((u, h), x)=\alpha(u, x) \cdot h$.

1 The author holds a National Science Foundation Postdoctoral Fellowship. 
Proposition 2.2. Let $B$ be a principal bundle over a topological group $G$. The action of $G$ on itself by left translation can be lifted to $E$ in $B$ if and only if $B$ is the product bundle.

Indeed, each orbit of $G$ in $E$ will be a cross-section. Since there are nontrivial bundles over almost all topological groups, this shows that the lifting in general is impossible. However, a measure of what stops the lifting will then be a measure of the nontrivialness of the bundle in this case. Similarly, in the case of Proposition 2.1 such a measure would tell us those bundles not obtained in the canonical way of factoring out a suitable isotropy group in a transitive action.

3. This section gives the statement and sketches the proof of the main theorem. We assume throughout this section that $B$ is a principal bundle with structural group $H$, a torus of dimension $m$. We also assume that $G$ is a semi-simple, compact, connected Lie group. To avoid coverings we suppose $G$ is simply connected. Nothing is lost in this last assumption as we have not demanded that the action of $G$ be effective.

THEOREM 3.1. Under the above hypothesis, if $B$ is paracompact and satisfies the first countability axiom then there is a bundle lifting of $(G, \bar{\alpha}) .^{2}$

LEMMA 3.2. In order that there be a bundle lifting under our hypothesis, it is sufficient that we have a bundle lifting over a fixed neighborhood $U$ of the identity in $G$.

This follows from the monodromy theorem $[1$, p. 49].

LEMMA 3.3. The mapping $\bar{\alpha}$ restricted to $U \times B$ where $U$ is a suitably chosen neighborhood of $e$ in $G$ can be lifted to a bundle map $\alpha: U \times E \rightarrow E$ such that $\alpha(e, x)=x, x \in E$.

Choose $U$ homeomorphic to a cube and apply the covering homotopy theorem, $[4$, p. 555]. (The paracompactness is needed here.)

Definition 3.1. Let $V$ be a neighborhood of $e$ in $G$ with $V^{3} \subset U$. We define the error function $\bar{f}: V \times V \times B \rightarrow H$ by the equation

$$
\alpha\left(u_{1} \cdot u_{2}, x\right)=\alpha\left(u_{1}, \alpha\left(u_{2}, x\right)\right) \cdot \bar{f}\left(u_{1}, u_{2} ; p(x)\right), \quad x \in E
$$

where $\alpha$ is given by Lemma 3.3. $\bar{f}$ is clearly continuous.

From the associative law we have

2 The author wishes to express his appreciation to Dr. R. S. Palais for pointing out that a hypothesis of simple connectivity is not needed. 


$$
\bar{f}\left(u_{1}, u_{2} ; u_{3} b\right)^{-1} \cdot \bar{f}\left(u_{2}, u_{3} ; b\right) \cdot \bar{f}\left(u_{1}, u_{2}, u_{3} ; b\right) \cdot \bar{f}\left(u_{1} \cdot u_{2}, u_{3} ; b\right)^{-1}=1,
$$

where we use the notation $u \cdot b=\bar{\alpha}(u, b)$. If $\bar{g}: V \times B \rightarrow H$ is a continuous map then we define a new covering $\alpha^{\prime}$ by

$$
\alpha^{\prime}(u, x)=\alpha(u, x) \cdot \bar{g}(u ; x) .
$$

In order that $\alpha^{\prime}$ give the action of a local group it is necessary and sufficient that we have

$$
\bar{f}\left(u_{1}, u_{2} ; b\right)=\bar{g}\left(u_{2} ; b\right) \cdot \bar{g}\left(u_{1} ; u_{2} \cdot b\right) \cdot \bar{g}\left(u_{1} \cdot u_{2} ; b\right)^{-1} \text {. }
$$

Taking $V$ contractible, we see that the map $\bar{f}$ is homotopic to the constant map $V \times V \times B \rightarrow 1 \in H$. Then there is a unique lifting of $\bar{f}$ to a map $f: V \times V \times B \rightarrow E^{m}$, euclidean $m$-space, satisfying $f\left(e, e, b_{0}\right)$ $=0$. Then $f$ will satisfy the equation (3.2) in $E^{m}$, (with the notation changed to additive notation). It is sufficient then to find $g: V \times B \rightarrow E^{m}$ satisfying (3.4) for $f$ in place of $\bar{f}$. We make now the following conversions.

$$
P\left(u_{1}, u_{2}, u_{3}\right)(b)=f\left(u_{1}^{-1} u_{2}, u_{2}^{-1} u_{3} ; u_{3}^{-1} \cdot b\right)
$$

then (3.2) becomes

$$
\begin{aligned}
P\left(u_{2}, u_{3}, u_{4}\right)(b)-P\left(u_{1}, u_{3}, u_{4}\right)(b)+P\left(u_{1}, u_{2}, u_{4}\right)(b) & \\
& -P\left(u_{1}, u_{2}, u_{3}\right)(b)=0 .
\end{aligned}
$$

Furthermore it is easily seen that

$$
\begin{aligned}
P\left(u u_{1}, u u_{2}, u u_{3}\right)(u b) & =P\left(u_{1}, u_{2}, u_{3}\right)(b), \\
P(e, e, e)(b) & =0 .
\end{aligned}
$$

We consider $P$ as a continuous function defined in a neighborhood of the diagonal $\Delta$ in $G \times G \times G$ and taking values in the topological group $\left(E^{m}\right)^{B}$ of continuous functions of $B$ into $E^{m}$ in the compact open topology. This group is easily seen to be an $A R$-space. Now suppose we have a continuous function $Q$ defined on some neighborhood of the diagonal in $G \times G$ and satisfying

$$
\begin{aligned}
P\left(u_{1}, u_{2}, u_{3}\right) & =Q\left(u_{2}, u_{3}\right)-Q\left(u_{1}, u_{3}\right)+Q\left(u_{1}, u_{2}\right), \\
Q\left(u u_{1}, u u_{2}\right)(u b) & =Q\left(u_{1}, u_{2}\right)(b) .
\end{aligned}
$$

Then if we define $g(u ; b)=Q(e, u)(u \cdot b) g$ will satisfy 2.4. $g$ is simultaneously continuous in both variables since $B$ satisfies the first axiom of countability [3, p. 103], and we could then conclude that the desired lifting exists.

We might as well assume that $P$ is defined on all of $G \times G \times G$ since 
$\left(E^{m}\right)^{B}$ is an $A R$ space. Now consider the sheaf of germs of continuous Alexander-Spanier cochains of degree $n$ with coefficients in $\left(E^{m}\right)^{B}$. This sequence of sheaves forms a soft, (since $\left(E^{m}\right)^{B}$ is an $A R$ space), acyclic resolution of the simple sheaf over $G$ with coefficients in $\left(E^{m}\right)^{B}$. By the Cartan uniqueness theorem, [2, p. 181] it follows that it yields the same cohomology for $G$ as does the usual Alexander Spanier cohomology. Since $H^{2}\left(G ;\left(E^{m}\right)^{B}\right)=0$ in this last theory it follows that there exists a continuous function $Q: G \times G \rightarrow\left(E^{m}\right)^{B}$ which satisfies (3.8) in a neighborhood of the diagonal $\Delta$ in $G \times G$. Since $G$ is compact we might as well assume this neighborhood homogeneous. Define

$$
Q\left(u_{1}, u_{2}\right)(b)=\int_{Q} Q\left(u u_{1}, u u_{2}\right)(u b) d u
$$

where the integral is the usual normalized Haar measure. $Q$ is the desired cochain. It also can be shown that the lifting is unique up to a bundle equivalence.

\section{REFERENCES}

1. C. Chevalley, Theory of Lie groups, Princeton University Press, 1946.

2. R. Godement, Theorie des faisceaux, Actualités Sci. Ind., 1958, p. 1252.

3. S. T. Hu, Homotopy theory, New York, Academic Press, 1959.

4. W. Huebsch, On the covering homotopy theorem, Ann. of Math. vol. 61 (1955) pp. $555-563$.

5. N. E. Steenrod, The topology of fibre bundles, Princeton University Press, 1951.

INSTITUTE FOR AdVANCED STUdy 